\title{
Perfil da condição bucal de idosas do Distrito Federal
}

\author{
Oral health status of elderly women \\ from the Brazilian Federal District
}

\author{
Ana M aria Costa ${ }^{1}$ \\ M aria do Carmo M achado Guimarães ${ }^{1}$ \\ Sérgio deFreitas Pedrosa ${ }^{2}$ \\ Otávio deTolêdo Nóbrega ${ }^{3}$ \\ Ana Cristina Barreto Bezerra ${ }^{2}$
}

${ }^{1}$ Departamento de

Odontologia, Faculdade de Ciências da Saúde,

Universidade Católica de

Brasília. EPCT QS 7, lote 1,

Águas Claras. 72030-170

Brasília DF.anam@ucb.br

${ }^{2}$ Departamento de

Odontologia, Faculdadede

Ciências da Saúde,

Universidade de Brasília.

${ }^{3}$ Faculdade de Ceilândia,

Universidade de Brasília.
Abstract Data from Brazilian researches that evaluated oral health of elderly peopleshow a worrisome situation. The purpose of this study was to estimatethefrequency of edentulism, analyzeboth the use and need profiles of prosthesis, calculate the DM FT index and check the condition of periodontal elderly residents in the Federal District. One hundred and forty women aged 60 years or above were examined. The condition of each prosthesis was evaluated to detect the presence of functional or aesthetic damage. Then, clinical examination was carried out to detect the number of decayed, missing or filled teeth in order to calculate the DM FT index. The periodontal examination included the index of visible plaque, bleeding index, measurement of clinical probing depth, measurement of clinical attachment level and teeth mobility. The results showed poor oral conditions of the patients. The rate of edentulism was high, the D M FT index was elevated (29.8) with a predominance of the extracted component $(87.1 \%)$ and periodontal condition was considered severe. From these data we can conclude that the oral condition of elderly represented in this study is precarious and reflects the need for health promotion and rehabilitation programs for this segment of the population.

Key words Elderly people, DM FT index, Edentulism
Resumo Os trabalhos realizados no Brasil a respeito das principais afecções bucais dos gerontes mostram situação preocupante. 0 objetivo deste estudo foi estimar a frequência de edentulismo, analisar o uso ea necessidade de prótese, calcular o índiceCPOD everificar a condição periodontal de idosas residentes no Distrito Federal. Cento equarenta mulheres com idade igual ou superior a sessenta anos foram examinadas. A condição de cada prótesefoi avaliada observando presença de prejuízo estético ou funcional. Em seguida, foi realizado exame clínico para detectar a presença de cárie, restaurações e dentes perdi dos para cálculo do índiceCPOD. 0 exameperiodontal incluiu a verificação do índice de placa visível, índice de sangramento gengival, medidas de profundidade de sondagem clínica, perda de inserção clínica e mobilidade dental de todos os dentes presentes. O s resultados revelaram péssimas condições bucais dos pacientes examinados. A taxa de edentulismo mostrou-se elevada, o índice CPOD foi alto $(29,8)$, com predomínio do componente extraído $(87,1 \%)$ e a condição periodontal foi considerada grave. A partir destes dados, pode-se concluir queo perfil da condição bucal das idosas representadas neste estudo é precário, o que reflete a necessidade de se elaborar programas depromoção de saúdeedereabilitação para este segmento da população.

Palavras-chave Idoso, Índice CPOD, Edentados 
Introdução

Levantamentos epidemiológicos são fundamentais para o estudo da distribuição de problemas de saúde na população e para a investigação dos fatores determinantes desta distribuição. Dados extraídos destes estudos possibilitam uma base para auxiliar a escolha das intervenções a serem implementadas em função da condição encontrada ${ }^{1}$.

Em se tratando do segmento da população com idade acima de sessenta anos, estudos de prevalência são escassos. Segundo a Fédération Dentaire Internationale ${ }^{2}$, são consideradas idosas as pessoas com mais de sessenta anos. 0 grupo de pessoas nesta faixa etária está crescendo de forma acelerada, com o aumento da expectativa de vida da população ${ }^{3}$.

Estima-se que $17 \%$ da população de países como Suécia e Japão apresentem idade superior a 65 anos. Já em países em desenvolvimento, este índice declina bastante 3 . No Brasil, a última pesquisa nacional de domicílios realizada pelo Instituto Brasileiro deGeografia e Estatística (IBGE) ${ }^{4}$ revelou que $9,7 \%$ dos indivíduos são idosos, 0 que equivale a aproximadamente 17,6 milhões depessoas. Contudo, algumas pesquisas estimam que, com o elevado ritmo de crescimento deste grupo etário, em 2025, os idosos representarão $15 \%$ da população brasileira ${ }^{5}$. Este fato evidencia a necessidade de maior preocupação com a saúde deste segmento etário, tendo em vista que doenças crônicas que requerem cuidados continuados são aspectos que podem acompanhar o envelhecimento.

No país, a situação que se observa no perfil bucal do idoso é precária. Estudos da década de noventa revelaram que cerca de $65 \%$ dos idosos mostravam ausência de dentes ${ }^{6,7}$. Trabalhos mais recentes reportam menores taxas de edentulismo; porém, as condições precárias na saúde bucal dos idosos quando se avalia o grande percentual de dentes extraídos no índice CPOD ainda são notórias ${ }^{8}$.

A odontogeriatria éuma especialidade recente da odontologia, que surgiu a partir destas novas necessidades da sociedade atual, relacionadas ao aumento da expectativa de vida da população ${ }^{8}$. Todavia, a odontologia geriátrica deve estar inserida em contexto integral de atenção à saúde. Considerando a diversidade e a complexidade do idoso, a atuação de uma equipe multiprofissional torna-se essencial, na medida em que analisa e integra conhecimentos específicos de áreas distintas com a finalidade comum de promover e manter a saúde do idoso.
A Universidade Católica de Brasília (UCBDF) vem desenvolvendo o Projeto para Promoção da Saúde dos I dosos, composto por professores, estudantes e profissionais de diferentes formações profissionais (nutrição, farmácia, medicina, educação física, fisioterapia, odontologia e biologia), que pesquisam aspectos da senescência humana9,10. 0 projeto tem seu foco principal na saúde de mulheres idosas. A justificativa de gênero éembasada no fato do envelhecimento populacional ser marcado por maior longevidade entre mulheres ${ }^{11}$.

A partir deste acompanhamento integral da saúde de idosas do Distrito Federal, foi observada a necessidade de se investigar o perfil da condição bucal dos indivíduos incluídos no projeto. Desta forma, o presente estudo teve como objetivo analisar o perfil de saúde bucal de idosas do Distrito Federal.

\section{Métodos}

\section{Descrição da amostra}

0 presente trabalho foi delineado como estudo transversal descritivo, com finalidade de traçar o perfil de saúde bucal demulheresidosas, com ênfasena frequência deedentulismo, na condição periodontal eno índiceCPOD dos sujeitos da pesquisa.

Esta pesquisa foi desenvolvida em parceria com o Programa de Pós-Graduação em Gerontologia da UCB-DF como segmento do Projeto para Promoção da Saúde dos I dosos. A casuística foi composta por amostra de conveniência, formada por mulheres não institucionalizadas constantes do banco de dados do projeto, com idade igual ou superior a sessenta anos, residentes na periferia do Distrito Federal, que atenderam a convite público para engajamento em projeto universitário envolvendo ações de promoção da saúde e acompanhamento ambulatorial voltadas ao controle da hipertensão arterial sistêmica, realizadas entre os meses de abril de 2006 e dezembro de $2007^{12}$. Desta forma, compôs-se um conjunto de regiões administrativas de procedência das idosas voluntárias, a saber: Candangolândia, Ceilândia, Núcleo Bandeirante, Recanto das Emas, Riacho Fundo, Samambaia e Taguatinga.

Quanto à condição socioeconômica da população idosa participante, estudos prévios demonstraram que a frequência conjunta de indivíduos que não possuem educação formal ou 
querelatam ensino fundamental incompleto che ga a mais de $60 \%$ deste segmento etário. Com relação à renda familiar mensal, aproximadamente uma em cada três dessas idosas apresenta renda igual ou inferior a um salário mínimo ${ }^{13,14}$

A execução desteestudo seguiu critérios nacionais para pesquisa em seres humanos, estabelecidos pela Resolução nº 196/96 do Conselho Nacional de Saúde, assim como pelo Código de Ética Profissional O dontológico, segundo Resolução CFO 179/91.

Todos os pacientes foram informados da natureza e objetivos da pesquisa e, após instrução e/ou leitura, foram convidados a assinar termo de consentimento livre esclarecido.

\section{Procedimentos clínicos}

O exameclínico inicial foi conduzido com espelho plano bucal eiluminação com foco de luz em equipo odontológico. Nesta primeira etapa, as pacientes foram examinadas para avaliar 0 uso e a condição da prótese, segundo critérios modificados de Colussi et al.8: ausência total de dentes em um arco sem a presença de prótese; ausência dental de um ou mais elementos; presença de próteses com comprometimento estético e/ou funcional (próteses quebradas, mal adaptadas, associadas a lesões na mucosa, com desgaste excessivo, sem retenção); presença de próteses satisfatórias ou presença de todos os dentes sem a necessidade de prótese.

As pacientes que apresentavam dentes foram submetidas a exame radiográfico periapical (Ektaspeed, Eastman Kodak Co, Rochester, USA) pela técnica do paralelismo e, em alguns casos, exameradiográfico interproximal. Logo após, foi iniciado novo exame clínico para avaliar o índice CPOD. Este índice é composto pelos seguintes fatores: dentes cariados $(C)$, perdidos $(P)$ e obturados ( 0 ). 0 exame clínico seguiu critérios do manual do M inistério da Saúde ${ }^{15}$, que considera o dente cariado quando há evidência clínica de esmalte socavado, existindo uma cavidade definida com descoloração ou opacidade ao longo das margens. Nas superfícies proximais de dentes posteriores, o diagnóstico clínico foi complementado com exameradiográfico interproximal. 0 dente foi considerado "obturado" quando se observou a reconstituição da cavidade com material restaurador direto ou indireto. Dentes com lesão de cárie secundária foram classificados como cariados.

Em seguida, foi realizada a avaliação periodontal de cada paciente, a qual incluiu a análise da documentação radiográfica periapical, índice de placa visível ${ }^{16}$, índice de sangramento gengival ${ }^{16}$, medidas de profundidade de sondagem, perda de inserção clínica e mobilidade dental de todos os dentes presentes ${ }^{17}$. As medidas de profundidade de sondagem enível de inserção clínica foram realizadas com sonda periodontal milimetrada estéril do tipo "M ichigan 0", com marcações de Williams (N eumar, São Paulo, SP, Brasil). As medidas foram realizadas em quatro sítios por dente: mesio-vestibular, disto-vestibular, lingual/palatina e vestibular.

0 diagnóstico da condição periodontal foi baseado na perda de inserção clínica, conforme descrito por Armitage ${ }^{18}$. A severidade da doença em cada dente baseou-se no sítio de maior perda de inserção, caracterizado da seguintemaneira: periodontiteleve: perda deinserção de 1 a $2 \mathrm{~mm}$; moderada: 3 a 4 mm; severa: maior ou igual a $5 \mathrm{~mm}$.

\section{Resultados}

Foram examinadas 140 idosas com idade média de 69,8 anos. A faixa etária variou de sessenta a 95 anos.

\section{Índice CPOD}

Os dados do CPOD e seus componentes encontram-se listados na Tabela 1.

$A$ análise dos dados mostrou que 0 índice CPOD atingiu valor igual a 29,8. A avaliação dos componentes evidenciou grande participação dos dentes extraídos (87,1\%).

A prevalência decárie deraiz foi de $19 \%$, com maior frequência em caninos e pré-molares inferiores. Ao considerar as restaurações radiculares, a prevalência aumentou para $24 \%$.

Uso e necessidade de prótese

0 percentual de edentulismo para os arcos superior e inferior encontram-se na Tabela 2. Os

Tabela 1. Índice CPOD e seus componentes.

\begin{tabular}{lrrr}
\hline CPOD e seus componentes & \multicolumn{1}{c}{ N } & \multicolumn{1}{c}{$\%$} & Média \\
\hline Cariado & 158 & 3,8 & 1,1 \\
Perdido & 3.640 & 87,1 & 26,0 \\
Obturado & 380 & 9,1 & 2,7 \\
Total & 4.178 & 100,0 & 29,8
\end{tabular}


dados refletem altas taxas de edentulismo total, principalmente na arcada superior $(75,8 \%)$.

Em relação ao uso e necessidade de próteses, os resultados apontados na Tabela 3 revelaram que a grande maioria de idosas usa algum tipo de prótese, sendo a prótese total a mais frequente no arco superior $(74,3 \%)$ e inferior $(47,1 \%)$. Vale ressaltar que muitas idosas não utilizam prótese inferior $(26,4 \%)$, apesar da necessidadee indicação.

Quando se analisa a Tabela 4, pode-se observar que grande parte dos trabalhos reabilitadores encontra-se em condição insatisfatória, independente do tipo de prótese.

A condição da prótese foi avaliada em relação à presença de prejuízos estético-funcionais. Dentre os problemas detectados em portadores de próteses superiores, a presença de lesões hiperplásicas devido à câmara desucção foi o maisfrequente $(75 \%)$, seguindo-se do desgaste acentuado (60\%), falta de retenção e/ou adaptação $(20 \%)$ e fraturas ( $18 \%)$. No caso da arcada inferior, o principal problema detectado foi a falta de retenção da prótese $(70 \%)$. Cabe lembrar que o mesmo aparelho protético, em alguns casos, apresentava mais de um defeito estético e/ou funcional.

Tabela 2. Distribuição do percentual de edentulismo.

\begin{tabular}{lrrrrrr}
\hline & \multicolumn{2}{c}{ Arco superior } & & \multicolumn{2}{c}{ Arco inferior } \\
\cline { 2 - 3 } \cline { 5 - 6 } \multicolumn{1}{c}{ Condição } & N & $\%$ & & N & $\%$ \\
\hline Edentada total & 106 & 75,8 & & 74 & 52,8 \\
Desdentadas parciais & 29 & 20,7 & & 60 & 42,9 \\
Arcada completa & 5 & 3,5 & & 6 & 4,3 \\
\hline
\end{tabular}

Tabela 3. Distribuição das idosas segundo o uso e a necessidade de prótese por arcada e tipo.

\begin{tabular}{|c|c|c|c|c|}
\hline & \multicolumn{2}{|c|}{ Arcada superior } & \multicolumn{2}{|c|}{ Arcada inferior } \\
\hline & $\mathrm{N}$ & $\%$ & $\mathrm{~N}$ & $\%$ \\
\hline Prótese total & 104 & 74,3 & 66 & 47,1 \\
\hline PPR * & 14 & 10,0 & 31 & 22,2 \\
\hline Prótese fixa & 4 & 2,8 & 0 & 0 \\
\hline \multicolumn{5}{|l|}{ Não usam } \\
\hline Indicado & 13 & 9,3 & 37 & 26,4 \\
\hline Não indicado & 5 & 3,6 & 6 & 4,3 \\
\hline Total & 140 & 100 & 140 & 100 \\
\hline
\end{tabular}

*PPR - Prótese parcial removível.

\section{Condição periodontal}

Ao se considerar a análise periodontal, a maioria das pacientes apresentou grau moderado ou severo da doença, com perda de inserção clínica elevada associada à presença de placa e sangramento gengival, que indicam sinais clínicos da inflamação dos sítios analisados (Tabela 5).

\section{Discussão}

Os trabalhos que envolvem pesquisas epidemiológicas possuem relevância na medida em que fornecem dados sobre prevalência e severidade das doenças. Além disso, esclarecem aspectos re lativos à etiologia e aos fatores determinantes do

Tabela 4. Distribuição das idosas segundo a condição da prótese (satisfatória ou insatisfatória).

\begin{tabular}{|c|c|c|c|c|}
\hline & \multicolumn{2}{|c|}{ Arcada superior } & \multicolumn{2}{|c|}{ Arcada inferior } \\
\hline & $\mathrm{N}$ & $\%$ & $\mathrm{~N}$ & $\%$ \\
\hline \multicolumn{5}{|l|}{ Prótese total } \\
\hline Satisfatória & 30 & 21,4 & 24 & 17,1 \\
\hline Insatisfatória & 74 & 52,9 & 42 & 30,0 \\
\hline \multicolumn{5}{|l|}{$\mathrm{PPR}^{*}$} \\
\hline Satisfatória & 3 & 2,2 & 7 & 5,0 \\
\hline Insatisfatória & 11 & 7,9 & 24 & 17,1 \\
\hline \multicolumn{5}{|l|}{ Prótese fixa } \\
\hline Satisfatória & 0 & 0 & 0 & 0 \\
\hline Insatisfatória & 4 & 2,8 & 0 & 0 \\
\hline Ausência de prótese & 18 & 12,8 & 43 & 30,8 \\
\hline Total & 140 & 100 & 140 & 100 \\
\hline
\end{tabular}

*PPR - Prótese parcial removível.

Tabela 5. Características periodontais das pacientes idosas: média da profundidade de sondagem clínica, média da perda de inserção clínica, média do índice de placa e média do índice gengival.

\begin{tabular}{lcc}
\hline \multicolumn{1}{c}{ Características } & $\begin{array}{c}\text { Gengivite } \\
\text { ou DP leve } \\
{ }^{*}(\mathrm{n}=22)\end{array}$ & $\begin{array}{c}\text { DP } \\
\text { moderada a } \\
\text { severa* }(n=41)\end{array}$ \\
\hline M édia sondagem & 1,79 & 2,21 \\
M édia perda de inserção & 0,75 & 5,63 \\
Índice de placa & 0,27 & 0,40 \\
Índice gengival & 0,23 & 0,67
\end{tabular}

DP: Doença periodontal. 
processo ${ }^{1}$. Em se tratando das afecções odontológicas que acompanham o processo de envelhecimento, são poucos os estudos que estimam prevalência de cárie, doença periodontal e edentulismo em indivíduos com idade acima de sessenta anos.

No Brasil, o Levantamento Epidemiológico em Saúde Bucal ${ }^{15}$, realizado em 1986, apesar de não contemplar a faixa etária acima de sessenta anos, divulgou situação crítica no grupo etário de cinquenta a 59 anos de idade. Os dados deste levantamento revelaram CPOD médio de 27,2 para esta faixa etária, com $86 \%$ de participação dos dentes extraídos, refletindo uma condição oral severamente insatisfatória naqueles indivíduos que atingiram a terceira idade. Desta for$\mathrm{ma}$, os resultados encontrados na presente pesquisa refletem o cenário nacional na medida em que também registram alto índice CPOD e alta taxa de dentes extraídos (Tabela 1). Ademais, nossos resultados corroboram que a situação de saúde bucal da presente geração de idosos não apresentou melhora em relação à daquela considerada no estudo de 1986. Os dados do mais recente levantamento epidemiológico bucal realizado no Brasil ${ }^{19}$ confirmam o CPOD elevado da população idosa, cujo valor atingiu 27,7 no grupo etário de 65 a 74 anos. 0 componenteperdido chegou a aproximadamente $93 \%$, enquanto os dentes obturados corresponderam a menos de $3 \%$ dos idosos pesquisados. Estes dados, assim como os encontrados na presente pesquisa (Tabela 1), refletem a falta de políticas preventivas de saúde destinada à população geriátrica para que este grupo mantenha seus dentes por toda a vida. Além disso, os dados retratam a falta de acesso a tratamentos restauradores pelos idosos, o que torna a extração o procedimento mais frequente para solucionar casos de dor.

Estudo efetuado na região de São Paulo re portou quea condição bucal de pacientesidososé crítica. O percentual deedêntulosindicou quemais da metadedos indivíduos perderam todososdentes ${ }^{6}$. Estes resultados são semelhantes aos aqui encontrados (Tabela 2). Sabe-se que a perda da dentição natural influi sobre diversos aspectos do organismo, dentre os quais o aspecto estético, a fonética e a função mastigatória. Estes problemas podem ser, em parte, solucionados pelo uso de prótese. Contudo, al guns estudos verificaram que o número deprótesesinsatisfatórias devido a problemasfuncionais e/ou estéticos éelevado $0^{20}$. Além disso, segundo Frare et al. ${ }^{7}$, a maior parte dos edêntulos não utiliza a prótese total inferior, alegando desconforto com a mesma. Este fato foi constatado também pelo presente trabalho, que mostrou 0 grande número de trabalhos reabilitadores insatisfatórios (Tabela 4) etambém a não utilização de prótese inferior por inúmeros indivíduos (Tabela 3), principalmente devido ao problema de retenção da mesma.

0 grave problema relacionado à perda dentária apontado na presente pesquisa podeestar relacionado à percepção dos idosos sobre sua saúde oral. Estudo realizado no Distrito Federal com pessoas da terceira idade institucionalizadas mostrou que a saúde oral é percebida com pouca importância entre os idosos. A perda dentária é vista como algo natural econsequenteda idade. Osidosos adaptam-se às suas limitações e aprendem a conviver com as sequelas das doenças por considerá-las normais ao processo de envelhecimento. Estefato refletea ausência deorientação apropriada voltada para o envelhecimento saudável ${ }^{21}$.

Outra condição frequentemente apontada em trabal hos é a alta ocorrência de cárie de raiz em indivíduos idosos ${ }^{8,22,23}$. Estudo em população idosa japonesa reportou que $39 \%$ dos indivíduos apresentaram uma ou mais raízes cariadas ${ }^{22}$, assim como o trabalho de Rins et al. ${ }^{23}$, que encontrou taxa de $31,8 \%$ de lesões cariosas radiculares entreidosos de São Paulo. O registro delesões de cárie de raiz verificado no presente estudo também se mostrou bastante alto. 0 aumento da frequência de lesões cariosas na raiz tem etiologia multifatorial, mas a exposição supragengival de superfícies radiculares é considerada prérequisito para a cárie radicular. A exposição frequentemente se deve à perda de aderência e à recessão associada à doença periodontal ou a seu tratamento ${ }^{24}$.

Em se tratando da doença periodontal, dados relacionados à idade acima de sessenta anos são escassos, embora a severidade e extensão desta afecção aumentecom a idade. 0 envel hecimento por si não implica maior suscetibilidade do indivíduo em desenvolver a doença periodontal, mas os efeitos cumulativos da doença no decorrer da vida podem explicar a maior prevalência da periodontite crônica em pessoas idosas ${ }^{25}$. Estudo realizado em Goiânia (GO) mostrou que $55 \%$ dos idosos institucionalizados exibiram presença de cál culo ${ }^{26}$. As condições periodontais encontradas por Rosa et al. ${ }^{6}$ também foram severas. Nos idosos em domicílios, metade dos sextantes examinados necessitavam de tratamento complexo (raspagem radicular e/ou tratamento cirúrgico). Já nas pessoas examinadas nas instituições, este percentual mostrou-se mais elevado. Os resultados da presente pesquisa revela- 
ram que grande parte das idosas dentadas apresentou doença periodontal crônica de grau moderado ou severo. Somente pequena parte da amostra obteve 0 diagnóstico de gengivite ou periodontite de grau leve, confirmando a alta prevalência da doença neste grupo etário, conforme mostra a literatura.

\section{Conclusões}

O perfil precário de saúde bucal dos idosos foi confirmado pelo presente trabalho. Os escassos estudos com esta faixa etária revelam elevado índice CPOD, grande percentual de dentes extraídos, muitas necessidades protético-reabilitadoras e péssima condição periodontal. Urge a busca de novas estratégias de prevenção econtrole do quadro atualmente instalado em indivíduos idosos. Sugere-seque ações específicas desaúdebucal para a terceira idade sejam inseridas o quanto antes nos programas de saúde já existentes, a fim de estimular a manutenção de condições odontológicas saudáveis por toda a vida e para superar, ao menos em parte, o crítico cenário atual.

\section{Colaboradores}

AM Costa e OT Nóbrega trabalharam na concepção do estudo e seu delineamento, na consecução da metodologia, na análisee interpretação dos resultados, na redação do manuscrito e na revisão crítica para aprovação final do artigo; MCM Guimarães, SF Pedrosa e ACB Bezerra participaram das etapas de análise dos dados e redação do manuscrito. 


\section{Referências}

1. Pereira MG. Indicadores de Saúde. In: Pereira MG. Epidemiologia teoria e prática. Rio de Janeiro: Guanabara Koogan; 1999. p. 49-73.

2. Fédération Dentaire Internationale. Oral needs of the elderly. Amsterdam: FDI; 1987.

3. Niessen LD. Geriatric dentistry in the next millennium: opportunities for leadership in oral health. Gerodontology 2000; 17(1):3-7.

4. Instituto Brasileiro de Geografia e Estatística. Dire toria de Pesquisas, coordenação de trabalho e rendimento, pesquisa nacional por amostra de domicílios 2004-2005. [site da Internet]. Disponível em: http:/ /ibge.gov.br

5. Barbosa AF, Barbosa AB. Odontologia geriátrica perspectivas atuais. J Bras Clin Odontol Int 2002; 6(33):231-234.

6. Rosa AGF, Fernandez RAC, Pinto VG, Ramos LR Condição de saúde bucal em pessoas de 60 anos ou mais no município de São Paulo. Rev. Saude Publica 1992; 26(3):155-160.

7. Frare SM, Limas A, Albarello FJ, Pedot G, Régio RAS. Terceira idade: quais os problemas bucais existentes? Rev Assoc Paul Cirur Dent 1997; 5(16):573-576.

8. Colussi CF, Freitas SFT, Calvo MCM. Perfil epidemiológico da cárie e do uso e necessidade de prótese na população idosa de Biguaçu, Santa Catarina. Rev. bras. epidemiol. 2004; 7(1):88-97.

9. Karnikowski M, Córdova C, Oliveira RJ, Karnikowski M GO, Nóbrega OT. Non-alcoholic fatty liver disease and metabolic syndrome in Brazilian middleaged and older adults. São Paulo Med J 2007; 125(6):333-337.

10. Colares FCJ, Silveira SRP, Silva APR, Córdova COA, Karnikowski MGO, Nóbrega OT. Ferro, folato e vitamina B12 - análise da ingestão e ocorrência de anemia em idosas. J Bras M ed 2008; 94(6):46-49.

11. Camarano AA, Kanso S, M ello JM. Como vive o idoso brasileiro? In: Camarano AA. Os novos idosos brasileiros. Muito além dos 60 ? Rio de Janeiro: IPEA; 2004.

12. Moraes CF, Souza ER, Souza VC, Medeiros EF, Gonçalves TF, Toledo JO, Karnikowski M, Gomes L, Karnikowski M G, Córdova C, Nóbrega OT. A common polymorphism in the renin angiotensin system is associated with differential outcome of antihypertensive pharmacotherapy prescribed to Brazilian older women. Clin Chim Acta 2008; 396(1-2):70-75.

13. Bortolon PC, Medeiros EFF, Naves JOS, Karnikowski MGO, Nóbrega OT. Análise do perfil de automedicação em mulheres idosas brasileiras. Cien Saude Colet 2008; 13(4):1219-1226.

14. Nóbrega OT, M elo GF, Karnikowski M GO. Pattern of drugs prescribed for community-residing middle-aged and older adults from the outskirts of Brasilia. Rev Bras Ciênc Farm 2005; 41(2):271-277.
15. Brasil. M inistério da Saúde. Levantamento Epidemiológico em Saúde Bucal: Brasil, Zona U rbana. Brasília: Ministério da Saúde;1986.

16. Ainamo J, Bay I. Problems and proposals for recording gingivitis and plaque. Int Dent J 1975; 25(4):229-235.

17. Bourgeois D, Bouchard P, Mattout C. Epidemiology of periodontal status in dentate adults in France, 2002-2003. J Periodont Res 2007; 42(3):219-227.

18. Armitage GC. Development of a classification system for periodontal diseases and conditions. Ann Periodontol 1999; 4(1):1-6.

19. Brasil. M inistério da Saúde. Condição de saúde bucal da população brasileira 2002-2003. Projeto SB Brasil 2003. Brasília: Ministério da Saúde; 2004.

20. Colussi CF, Freitas SFT. Aspectos epidemiológicos da saúde bucal do idoso no Brasil. Cad Saude Publica 2002; 18(5):1313-1320.

21. Somchinda A, Fernandes FC. Saúde e qualidade de vida na terceira idade: uma introspecção dos idosos institucionalizados [monografia de especialização]. Brasília (DF): Associação Brasileira de Odontologia; 2003.

22. Imazato S, Ikebe K, Nokubi T, Ebisu S, Walls AW. Prevalence of root caries in a select population of older adults in Japan. J Oral Rehabil 2006; 33(2):137143.

23. Reis SCGB, Higino M ASP, M elo HMD, Freire M CM. Condição de saúde bucal de idosos institucionalizados em Goiânia-GO, 2003. Rev. bras. epidemiol. 2005; 8(1):67-73.

24. Beck J. The epidemiology of root surface caries. J Dent Res 1990; 69(5):1216-1221.

25. Papapanou PN, Lindhe J. Epidemiologia das doenças periodontais. In: Lindhe F, Karring T, Lang NP, organizadores. Tratado de periodontia clínica e implantodontia oral. Rio de Janeiro: Guanabara Koogan; 1999. p. 49-73.

26. Rihs LB, Souza MLR, Wada RS. Prevalência de cárie radicular em adultos e idosos na região sudeste do estado de São Paulo, Brasil. Cad Saude Publica 2005; 21(1):311-316.

Artigo apresentado em 21/05/2008

A provado em 21/08/2008

Versão final apresentada em 31/03/2009 